\title{
Constitutive Model of Shape Memory Alloy Considering the Effect of Martensite Plasticity for Biomedical Applications
}

\author{
Yanping Wang ${ }^{1, a^{*}}$ \\ ${ }^{1}$ College of Medicine, Xi'an International University, Xi'an, Shaanxi, China \\ a375031254@qq.com
}

Keywords: Shape memory alloys; Super-elasticity; Constitutive law; Plasticity

\begin{abstract}
This paper focuses on the super-elastic characterization of Ni-based shape memory alloys for biomedical applications. Shape memory alloy (SMA) have presented excellent characterization in biomedical application fields, such as high corrosion resistance, bio-compatible, non-magnetic, and the unique physical properties with the capability to replicate those of human tissues and bones, and so on. In this study, a shape memory alloy (SMA) constitutive model is proposed that is capable of describing SMA super-elastic features and the plasticity effects. Finally, the analyzed results show good ability to predict y the experimental data.
\end{abstract}

\section{Introduction}

SMAs have been used in many fields for medical applications including stents, endodontics, sutures, medical tweezers, anchors for attaching tendon to bone, implants, aneurism treatments, eyeglass frames and guide wires and other medical devices and equipments in many fields including neurology, orthopaedics, cardiology and interventional radiology [1].

Some works are focused on the implementation of constitutive model of SMA into finite element package $[2,4]$. However, there are very few constitutive models considering the plastic deformation of the martensite under the conditions of stress-induced martensite transformation. Recently, Kan and Yan [5-6] developed a temperature-dependent three-dimensional phenomenological constitutive model considering the local plastic yield of martensite under high stress, and then successfully implemented into a finite element package ABAQUS.

In this work, a temperature-independent phenomenological constitutive model is proposed to describe the thermo-mechanical deformation of NiTi alloy combining superelastic and plasticity features. The prediction capability of the proposed model is verified by the experimental results. Finally, the numerical example is given to verify the validity of the implementation.

\section{Constitutive Model}

Generalized plasticity is firstly employed by Lubliner and Auricchio[2] to depicts the thermo-mechanical responses of shape memory alloys. With infinitesimal strain assumption, the additively decomposition of the total strain $\overline{\boldsymbol{\varepsilon}}$ into an elastic strain $\overline{\boldsymbol{\varepsilon}}_{\mathrm{e}}$ and an inelastic strain $\overline{\boldsymbol{\varepsilon}}_{\text {in }}$ yields:

$$
\overline{\boldsymbol{\varepsilon}}=\overline{\boldsymbol{\varepsilon}}_{\mathrm{e}}+\overline{\boldsymbol{\varepsilon}}_{\text {in }}
$$

Based on the assumption of small deformation, the total inelastic strain $\overline{\boldsymbol{\varepsilon}}_{\text {in }}$ is the stress-induced martensitic transformation $\overline{\boldsymbol{\varepsilon}}_{\mathrm{t}}$ and the plasticity transformation $\overline{\boldsymbol{\varepsilon}}_{\mathrm{p}}$. Therefore, the total inelastic strain $\overline{\boldsymbol{\varepsilon}}_{\text {in }}$ is expressed as:

$$
\overline{\boldsymbol{\varepsilon}}=\overline{\boldsymbol{\varepsilon}}_{\mathrm{e}}+\overline{\boldsymbol{\varepsilon}}_{\mathrm{in}}=\overline{\boldsymbol{\varepsilon}}_{\mathrm{e}}+\overline{\boldsymbol{\varepsilon}}_{\mathrm{t}}+\overline{\boldsymbol{\varepsilon}}_{\mathrm{p}}
$$

By discretizing the equation, the expression is as follows:

$$
\Delta \overline{\boldsymbol{\varepsilon}}_{\mathrm{in}}=\Delta \overline{\boldsymbol{\varepsilon}}_{\mathrm{t}}+\Delta \overline{\boldsymbol{\varepsilon}}_{\mathrm{p}}
$$




$$
\Delta \overline{\boldsymbol{\varepsilon}}_{\mathrm{e}}=\Delta \overline{\boldsymbol{\varepsilon}}-\Delta \overline{\boldsymbol{\varepsilon}}_{\mathrm{t}}-\Delta \overline{\boldsymbol{\varepsilon}}_{\mathrm{p}}
$$

The stress-induced martensitic transformation and its reverse transformation which can be defined by the volume fraction of martensite as the internal variable $\bar{\xi}$. The elastic stress-strain relation can be then expressed as:

$$
\overline{\boldsymbol{\sigma}}=\overline{\boldsymbol{\sigma}}^{*}-\overline{\mathbf{D}}^{\mathrm{e}}(\bar{\xi}): \Delta \overline{\boldsymbol{\varepsilon}}_{\text {in }}
$$

where $\overline{\mathbf{D}}^{\mathrm{e}}(\bar{\xi})$ is the equivalent elastic tensor, and $\overline{\mathbf{D}}^{\mathrm{e}}(\bar{\xi})=\left[(1-\bar{\xi}) \overline{\mathbf{D}}_{A}^{-1}+\bar{\xi} \overline{\mathbf{D}}_{M}^{-1}\right]^{-1}$.

where $\overline{\mathbf{D}}_{A}$ and $\overline{\mathbf{D}}_{M}$ are the elastic tensor of austenite and martensite, respectively. Considering the deviatoric stress of the above equation, it can be gotten $\overline{\mathbf{D}}^{\mathrm{e}}: \Delta \overline{\boldsymbol{\varepsilon}}_{\text {in }}=2 \bar{G} \Delta \overline{\boldsymbol{\varepsilon}}_{\text {in }}$, and:

$\overline{\mathbf{S}}=\overline{\mathbf{S}}^{*}-2 \bar{G} \Delta \overline{\boldsymbol{\varepsilon}}_{\text {in }}$

where $\bar{G}$ is the shear elastic modulus, and $\overline{\mathbf{s}}^{*}$ is the deviatoric stress of $\overline{\boldsymbol{\sigma}}^{*}$.

As mentioned in the references [7, 8], some phase transformations are pressure-dependent. To model such an effect, the Von-Mises-typed transformation surfaces are introduced:

$\bar{F}(\overline{\boldsymbol{\sigma}}, \bar{q})=\bar{\sigma}_{\mathrm{eq}}-\bar{\sigma}_{\mathrm{y}}(\bar{q})=0$

where $\bar{\sigma}_{\text {eq }}=\left[\frac{3}{2} \overline{\mathbf{S}}: \overline{\mathbf{S}}\right]^{\frac{1}{2}}$ is the von Mises equivalent stress. The phase transformation to describe the forward transformation and its reverse transformation are introduced as follows:

$\bar{F}_{\mathrm{AM}}(\overline{\boldsymbol{\sigma}}, \bar{\xi})=\bar{\sigma}_{\mathrm{eq}}-\sigma_{s, T}^{\mathrm{AM}}(\bar{\xi})=0$, forward transformation

$\bar{F}_{\mathrm{MA}}(\overline{\boldsymbol{\sigma}}, \bar{\xi})=\bar{\sigma}_{\mathrm{eq}}-\sigma_{s, T}^{\mathrm{MA}}(\bar{\xi})=0$, reverse transformation

where, $\sigma_{s, T}^{\mathrm{AM}}$ and $\sigma_{s, T}^{\mathrm{MA}}$ are the start stresses of the forward transformation and the reverse transformation, respectively.

In this paper, the reversible martensite volume fraction $\bar{\xi}$ is related to the transformation strain $\bar{\varepsilon}_{\mathrm{t}}$ by the following expression:

$\bar{\xi}=\bar{\varepsilon}_{\mathrm{t}} / \varepsilon_{\mathrm{L}}$

in which, $\varepsilon_{\mathrm{L}}$ is the maximum phase transformation strain, which is determined by the experiment.

Similar to the normality rule of the plasticity strain, the transformation strain rates obey the normality rule,

$\Delta \overline{\boldsymbol{\varepsilon}}_{\mathrm{t}}^{\mathrm{AM}}=\sqrt{\frac{3}{2}} \varepsilon_{\mathrm{L}} \Delta \bar{\xi}_{\overline{\mathbf{n}}} \overline{\mathrm{AM}}_{\mathrm{AM}}, \Delta \bar{\xi}>0$, forward phase transformation

$\Delta \boldsymbol{\varepsilon}_{\mathrm{t}}^{\mathrm{MA}}=\sqrt{\frac{3}{2}} \varepsilon_{\mathrm{L}} \Delta \bar{\xi} \overline{\mathbf{n}}_{\mathrm{MA}}, \Delta \bar{\xi}<0$, reverse phase transformation

with

$$
\overline{\mathbf{n}}_{\mathrm{AM}}=\frac{\bar{\partial} \bar{F}_{\mathrm{AM}}(\overline{\boldsymbol{\sigma}}, \bar{\xi})}{\bar{\partial} \overline{\boldsymbol{\sigma}}}=\sqrt{\frac{3}{2}} \frac{\overline{\mathbf{s}}}{\bar{\sigma}_{\mathrm{eq}}}
$$




$$
\overline{\mathbf{n}}_{\mathrm{MA}}=\frac{\bar{\partial} \bar{F}_{\mathrm{MA}}(\overline{\boldsymbol{\sigma}}, \bar{\xi})}{\bar{\partial} \overline{\boldsymbol{\sigma}}}=\sqrt{\frac{3}{2}} \frac{\overline{\mathbf{s}}}{\bar{\sigma}_{\mathrm{eq}}}
$$

In this paper, the phase transformation, austenite yield and martensite yield behavior under different temperature are considered in the process of implicit stress integral solution. Considering the implicit stress integration process of the phase change behavior, Equation (6) can be further expressed as,

$$
\overline{\mathbf{S}}=\overline{\mathbf{S}}^{*}-2 \bar{G} \Delta \overline{\boldsymbol{\varepsilon}}_{\mathrm{t}}
$$

By Eqs. (10a-b), it can be obtained:

$\Delta \overline{\boldsymbol{\varepsilon}}_{\mathrm{t}}^{\mathrm{AM}}=\sqrt{\frac{3}{2}} \varepsilon_{\mathrm{L}} \Delta \bar{\xi}_{\overline{\mathbf{n}}} \overline{\mathrm{AM}}_{\mathrm{I}}, \Delta \bar{\xi}>0$, forward phase transformation

$$
\Delta \overline{\boldsymbol{\varepsilon}}_{\mathrm{t}}^{-\mathrm{MA}}=\sqrt{\frac{3}{2}} \varepsilon_{\mathrm{L}} \Delta \bar{\xi}_{\mathbf{n}_{\mathrm{MA}}}^{-} \Delta \bar{\xi}<0, \text { reverse phase transformation }
$$

in which, $\overline{\mathbf{n}}_{\mathrm{AM}}=\overline{\mathbf{n}}_{\mathrm{MA}}=\overline{\mathbf{n}}_{\mathrm{t}}=\sqrt{\frac{3}{2}} \frac{\overline{\mathbf{s}}}{\bar{\sigma}_{\mathrm{eq}}}$

If the loading is continued up to exceed the martensite plastic yield stress after finishing its stress-induced phase transformation, it will occur the martensite plastic deformationf. In this research, it is assumed that the plastic yield behavior of martensite subject to von Mises yield criterion, and the plastic yield condition can be expressed as:

$$
\bar{F}_{\mathrm{Mp}}(\overline{\boldsymbol{\sigma}}, \bar{p})=\bar{\sigma}_{\mathrm{eq}}-\sigma_{\mathrm{y}}^{\mathrm{M}}(\bar{p})=0
$$

in which, the internal variable $\bar{p}$ is the cumulative plastic strain as:

$$
\Delta \bar{p}=\left[\frac{2}{3} \Delta \overline{\boldsymbol{\varepsilon}}_{\mathrm{p}}: \Delta \overline{\boldsymbol{\varepsilon}}_{\mathrm{p}}\right]^{\frac{1}{2}}
$$

and $\sigma_{\mathrm{y}}^{\mathrm{M}}$ is the plastic yield stress martensite.

The plastic strain rate can be expressed as:

$\Delta \overline{\boldsymbol{\varepsilon}}_{\mathrm{p}}=\Delta \overline{\boldsymbol{\varepsilon}}_{\mathrm{p}}^{\mathrm{M}}=\Delta \lambda_{\mathrm{M}} \frac{\partial \overline{\boldsymbol{F}}_{\mathrm{Mp}}(\overline{\boldsymbol{\sigma}}, \overline{\dot{\xi}})}{\partial \overline{\boldsymbol{\sigma}}}=\sqrt{\frac{3}{2}} \Delta \bar{p} \overline{\mathbf{n}}_{\mathrm{M}}$, martensite plastic yield

$\lambda_{\mathrm{M}}$ is the plastic multipliers of the martensite plastic yield. $\overline{\mathbf{n}}_{\mathrm{M}}$ is the direction vector of the martensite plastic yield,

$$
\overline{\mathbf{n}}_{\mathrm{A}}=\overline{\mathbf{n}}_{\mathrm{M}}=\sqrt{\frac{3}{2}} \frac{\overline{\mathbf{s}}}{\bar{\sigma}_{\mathrm{eq}}}
$$

\section{Verification of Proposed Model}

The results of the phase transformation simulations by the proposed model are compared with the experimental data by Kang [9]. To verify the proposed model, the super-elastic stress-strain curves at different temperatures are simulated using the parameters listed as follows to identify the parameters.

$E_{\mathrm{A}}^{T_{0}}=41.0 \mathrm{GPa}, E_{\mathrm{M}}^{T_{0}}=37.0 \mathrm{GPa} ; v_{A}=v_{M}=0.33 ; C_{A M}=8.0 \mathrm{MPa} / \mathrm{K}, C_{M A}=8.8 \mathrm{MPa} / \mathrm{K} ; k=0.16 ; \sigma_{s, T 0}^{A M}$ $=353.0 \mathrm{MPa}, \sigma_{f, T 0}^{A M}=381.0 \mathrm{MPa}, \sigma_{s, T 0}^{M A}=141.0 \mathrm{MPa}, \sigma_{f, T 0}^{M A}=122.0 \mathrm{MPa} ; \varepsilon_{L}=0.035 ; \quad T_{0}=295 \mathrm{~K} ; h_{\mathrm{M}}^{\mathrm{p}}=$ 6.7GPa. 


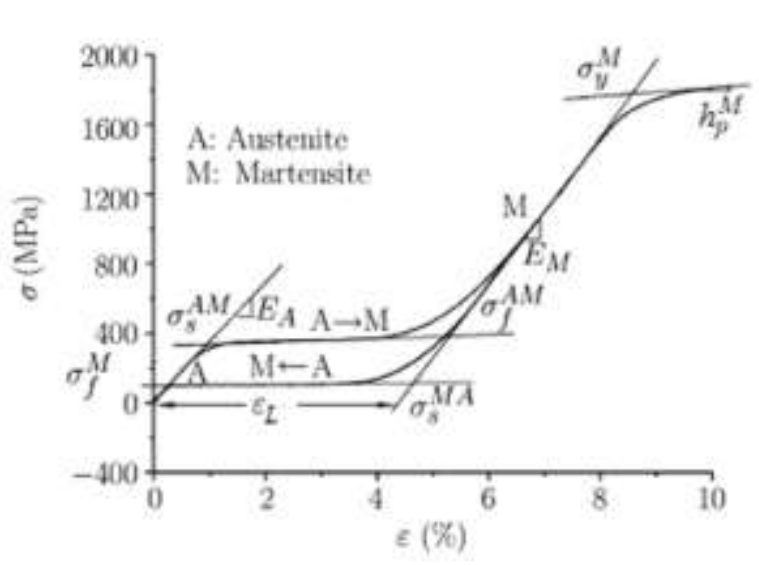

(a)

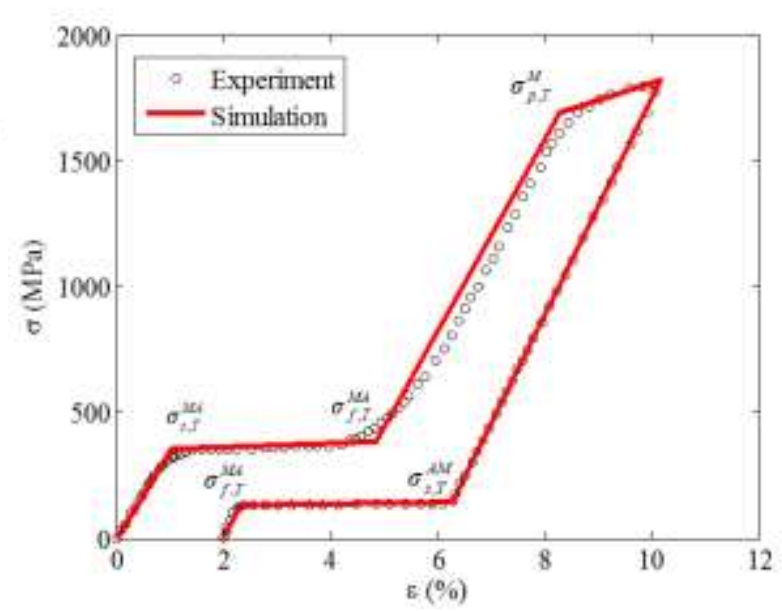

(b)

Figure 1. Uniaxial tension and unloading of NiTi shape memory alloy: (a) SMA phase transformation and plasticity behavior diagram; (b) Stress-strain curves during tension and unloading

The uniaxial tension and unloading case of the material is calculated based on the proposed model. The simulation results are shown in Fig.1(b). It can be seen that the stress-induced martensitic transformation occurs. With higher applied peak strain, an apparent residual strain can be observed which is caused by the plastic deformation of the martensite. The simulated results show the good agreement with the experimental ones. The results show that the proposed constitutive model can predict the thermodynamic behavior in the super-elastic NiTi alloy. It can be predicted that the model can also give a reasonable prediction results for other working conditions and other experimental results in the temperature range.

\section{References}

[1] Jani J.M., Leary M., Subic A. and Gibson M. A. A review of shape memory alloy research, applications and opportunities, Mater Design. Vol. 56 (2014), p.1078-1113.

[2] F. Auricchio and R.L. Taylor, Shape-memory alloy modeling and numerical simulations of the finite-strain superelastic behavior, Comput Method Appl Mech Eng. Vol. 143 (1997), p.175-194.

[3] F. Auricchio, A robust integration algorithm for a finite strain shape memory alloy superelastic model, Int. J. Plasticity. Vol. 17 (2001), p.971-990.

[4] N. Rebelo, M. Hsu and H. Foadian, Simulation of super-elastic alloys behavior with abaqus. Proc. International Conference on Shape memory and Super-elastic Technologies. SMST, 2000, Pacific Grove (USA, 2001) 457-469.

[5] L. Saint-Sulpice, S.A. Chirani, S Calloch. A 3D superelastic model for shape memory alloys taking into account progressive strain under cyclic loadings, Mech. Mater. Vol. 41 (2009), p.12-26.

[6] Ivshin Y., Pence T. A thermomechanical model for a one variant shape memory material, J. Intel Mat. Syst Str. Vol. 5 (1994), p.455-473.

[7] Liang C., Rogers C. One-dimensional thermomechanical constitutive relations for shape memory materials. J. Intel Mat. Syst Str. Vol. 1 (1990), p.207-234.

[8] Auricchio F., Sacco E. A one-dimensional model for superelastic shape memory alloys with diffeent elastic properties between austenite and martensite, Int. J Nonlin. Mech. Vol. 32 (1997), p.1101-1114.

[9] Kang G.Z., Kan Q.H., Qian L.M. and Liu Y.J. Ratchetting deformation of super-elastic and shape-memory NiTi alloys, Mech. Mater. Vol. 41 (2009), p.139-153. 